\title{
Radiative Levitation in Massive Stars: A self-consistent approach
}

\author{
Durand D'souza and Achim Weiss \\ Max Planck Institute for Astrophysics, Garching, Germany \\ email: durand@mpa-garching.mpg.de
}

\begin{abstract}
In B stars, the transportation of metals such as iron due to radiative levitation is thought to trigger sub-surface convection, which may lead to significant stellar pulsations through the iron-induced kappa mechanism (Pamyatnykh et al. 2004). The main goal of this work is to model the evolution of these stars by treating radiative levitation in an accurate and efficient manner in order to enable rapid asteroseismic analysis using the Opacity Project data and codes (Seaton 2005) with the GARching STellar Evolution Code (Weiss \& Schlattl 2008).
\end{abstract}

Keywords. stars: abundances, stars: evolution, stars: interiors, stars: oscillations (including pulsations), diffusion, atomic processes

Asteroseismic diagnostics of massive stars requires the consideration of the various pulsation modes in detail. In massive $\mathrm{O}$ stars, the opacity peak in iron at $\log (T) \approx 5.3$ and the large luminosities involved naturally give rise to a sub-surface convection zone in the radiative envelope of the star. These sub-surface convective zones have also been linked to non-radial pulsations, such as in $\beta$ Cephei and slowly pulsating B stars (Cantiello et al. 2009). In less massive B stars with similar metallicity, the reduced luminosity means that a larger opacity is necessary to trigger convection. This increase is hypothesised to be due to radiative levitation of elements, especially iron.

For consistency, we utilise the Opacity Project (OP) data and codes (Seaton 2005) within the GARching STellar Evolution Code (Weiss \& Schlattl 2008) to calculate the Rosseland mean opacity at each point in the star on-the-fly. Different methods of calculating the Rosseland mean opacity produce somewhat different results (Fig. 1). As the Opacity Project does not consider the effects of high temperatures and molecular opacities at low temperatures, we need to interpolate with the OPAL tables within the relevant temperature domain. Composition changes due to diffusion and radiative acceleration, amongst other processes, are treated by solving the Burgers equations exactly (Thoul et al. 1994). The radiative acceleration profile for a $5 M_{\odot}$ star may be seen in Fig. 2. We intend to apply the Single-Valued Parameter (SVP) approximation developed by LeBlanc \& Alecian (2004), which has been shown to produce similar radiative acceleration profiles to exact methods at a substantially reduced computational cost.

\section{References}

Cantiello, M., Langer, N., Brott, I., et al. 2009, A\& A 499, 279

LeBlanc, F. \& Alecian, G. 2004, MNRAS 352, 1329

Pamyatnykh, A. A., Handler, G., \& Dziembowski, W. A. 2004, MNRAS 350, 1022

Seaton, M. J. 2005, MNRAS 362, L1

Thoul, A. A., Bahcall, J. N., \& Loeb, A. 1994, ApJ 421, 828

Weiss, A. \& Schlattl, H. 2008, Ap\&SS 316, 99 


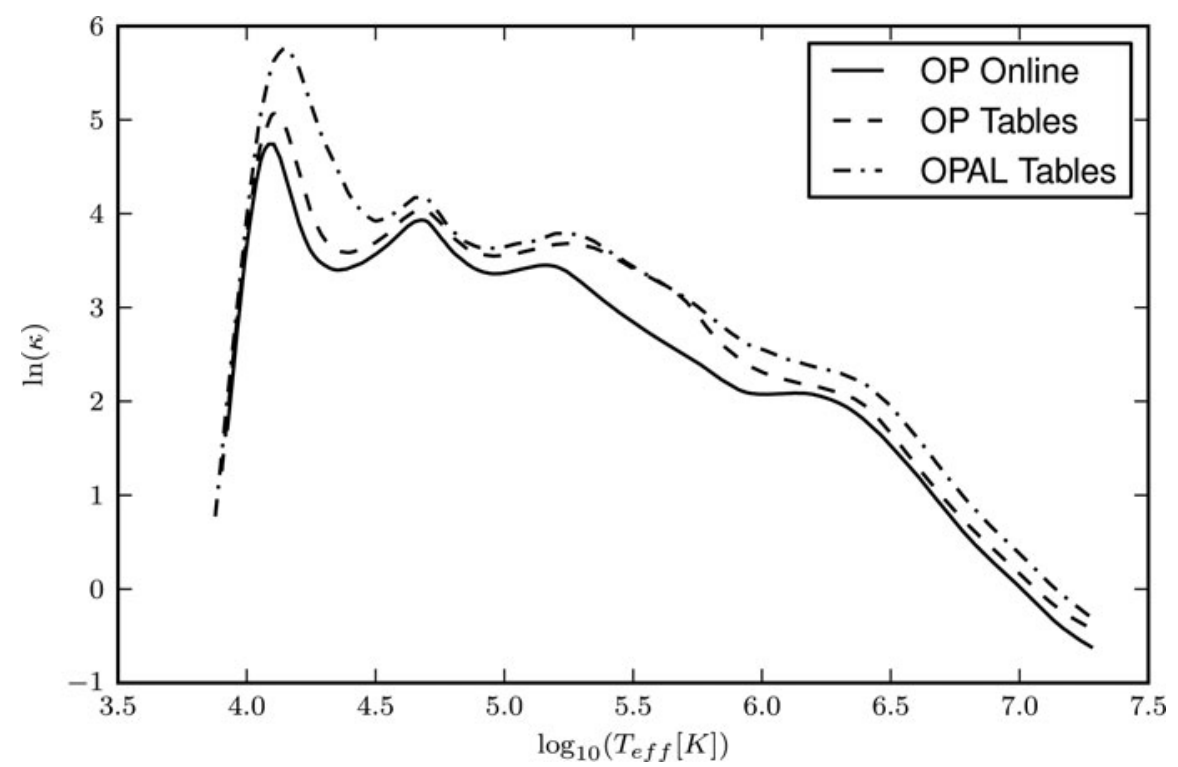

Figure 1. Differences between methods used to calculate the Rosseland mean opacity can lead to different results. In this plot, Rosseland mean opacities from on-the-fly calculations (OP Online) are compared with those interpolated from precomputed tables (OP Tables and OPAL Tables) for a $1.7 M_{\odot}$ star. While the Online version is self-consistent, it does not include modifications for high temperatures or for low temperatures, where molecular opacities play a big role.

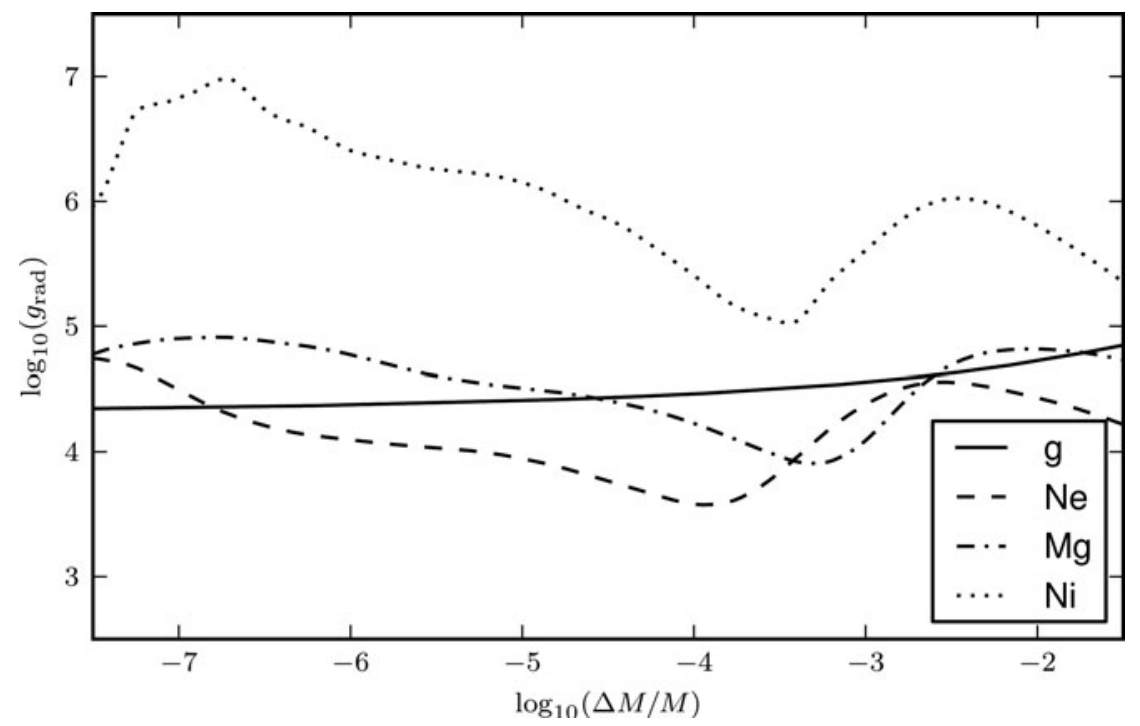

Figure 2. A plot showing the difference in radiative acceleration behaviour between various elements as a function of position in the star. The solid line represents gravitational acceleration. Neon has a radiative acceleration profile that is generally weaker than gravitational acceleration so it will gravitationally settle while Nickel will radiatively levitate. In general, higher mass stars have higher radiative accelerations due to the larger contribution from the radiation field. 